\title{
Catalytically Enhanced Cas9 through Directed Protein Evolution
}

Travis H. Hand ${ }^{1 \#}$, Mitchell O. Roth ${ }^{1 \#}$, Chardasia L. Smith ${ }^{2}$, Emily Shiel ${ }^{2}$, Kyle N. Klein ${ }^{3}$ and David M. Gilbert ${ }^{3}$, and Hong $\mathrm{Li}^{1,2 *}$

${ }^{1}$ Institute of Molecular Biophysics, Florida State University, Tallahassee, FL 32306, USA

${ }^{2}$ Department of Chemistry and Biochemistry, Florida State University, Tallahassee, FL 32306, USA.

${ }^{3}$ Department of Biological Sciences, Florida State University, Tallahassee, FL 32306, USA.

\#Equal contributing authors

*Corresponding author: hong.li@fsu.edu 
Abstract. The Clustered Regularly Interspaced Short Palindromic Repeat (CRISPR)-Cas9 system has found widespread applications in genome manipulations due to its simplicity and effectiveness. Significant efforts in enzyme engineering have been made to improve the CRISPR-Cas9 systems beyond their natural power with additional functionalities such as DNA modification, transcriptional regulation, and high target selectivity ${ }^{1-10}$. Relatively less attention, however, has been paid to improving the catalytic efficiency of CRISPRCas9. Increased catalytic efficiency may be desired in applications where the currently available CRISPR-Cas9 tools are either ineffective ${ }^{4,11-14}$ or of low efficiency such as with type II-C Cas $9^{15-18}$ or in non-mammals ${ }^{19,20}$. We describe a directed protein evolution method that enables selection of catalytically enhanced CRISPR-Cas9 variants (CECas9). We demonstrate the effectiveness of this method with a previously characterized Type IIC Cas9 from Acidothermus cellulolyticus (AceCas9) with up to 4-fold improvement of in vitro catalytic efficiency, as well as the widely used Streptococcus pyogenes Cas9 (SpyCas9), which showed a 2-fold increase in homology directed repair (HDR)-based gene insertion in human colon cancer cells.

Functional CRISPR-Cas9 enzymes are composed of a Cas9 protein, a CRISPR RNA (crRNA) and a trans-activating crRNA (tracrRNA). The crRNA and tracrRNA can often be replaced by a chimeric single-guide RNA (sgRNA) ${ }^{21}$. The sgRNA or crRNA contains a complementary region that base pairs with the target DNA (protospacer). Cas9 possesses multiple domains: a nucleic acid recognition domain (REC), a PAM (protospacer adjacent motif)-interacting domain (PID), a RuvC nuclease domain and an $\mathrm{HNH}$ nuclease domain (Figure 1A). Cas9 searches for and initially locates a PAM by its PID, which leads 
to unwinding of the protospacer and formation of the DNA-sgRNA heteroduplex, or Rloop. Finally, the HNH domain cleaves the target strand while the RuvC domain cleaves the non-target strand ${ }^{22-24}$. Previous biochemical and biophysical studies on SpyCas9 showed that it is essentially a single turnover enzyme with the rate-limiting step at DNA binding $^{25}$ or R-loop formation ${ }^{26}$. Importantly, DNA cleavage efficiency scales with sampling of the $\mathrm{HNH}$ domain conformation; a correct conformation enhances the cleavage activity of the RuvC domain ${ }^{27}$. This allostery between $\mathrm{HNH}$ and RuvC is believed to result from the hinge comprising two helices that connect RuvC-III to $\mathrm{HNH}^{21,27-32}$. Mutations that disrupt one of the two hinge helices lowered the DNA cleavage activity of SpyCas $9^{27}$. These detailed mechanistic studies suggest that the allosteric hinge is also a potential target for engineering CECas $9^{33}$. However, protein engineering by rational design to select for CECas9 variants would be laborious and have limited chances of successes.

We previously described a toxicity-based bacterial survival assay that is amenable for a library-based directed protein evolution selection ${ }^{34,35}$. In this approach, a library of plasmids encoding a collection of Cas9 variants is transformed into bacterial cells harboring a plasmid expressing the toxic protein $\mathrm{ccdB}$ under the induction by arabinose. In presence of arabinose, cells transformed with the Cas9 variants capable of cleaving the ccdB-encoding plasmid would grow whereas those containing the variants incapable of cleaving the ccdB-encoding plasmid would not. Thus, the survival cell colonies would allow identification of the Cas9 variants that cleave the ccdB-encoding plasmid. To identify CECas9 variants, we need to program the ccdB-encoding plasmid with a protospacer that is not cleaved by the wild-type Cas9. 
AceCas 9 was previously shown to be most efficient in cleaving protospacers with 24 nucleotide (nt) of spacer complementarity ${ }^{36}$. The rate of single-turnover cleavage of the 24-nt protospacer is 4 -fold that of the 20 -nt protospacer on supercoiled plasmid DNA. In bacterial cells, while AceCas9 targeting a 24-nt protospacer causes near one hundred percent survival in the ccdB-based cell survival assay, that targeting a 20 -nt protospacer has less than $0.2 \%$ rate of survival (Figure S1). In a typical transformation experiment, therefore, no surviving colonies would be observed when the wild-type AceCas9 is introduced to electro-competent $E$. coli cells harboring the ccdB-plasmid with a 20-nt protospacer ${ }^{35,36}$. We took advantage of this selection feature in identifying AceCECas9. We hypothesized that the allosteric hinge can be engineered to allow a faster $\mathrm{HNH}$ conformational sampling, which leads to faster RuvC cleavage, resulting in more efficient formation of double-stranded breaks. To this end, we generated a library of the hinge variants through error-prone Polymerase Chain Reaction (PCR) (Figure 1A).

In contrast to negligible growth of cells containing the ccdB plasmid transformed with a 20-nt protospacer wild-type AceCas9, those transformed with the hinge library showed significant growth. The survival colonies were then pooled, and their plasmid DNA were extracted for a second round of selection. The selection cycle was repeated until the rate of survival per microgram of DNA no longer increased. The DNA pool from each selection cycle was subjected to Next Generation Sequencing (NGS) and plasmid DNA from 20 individual colonies of the final cycle were also isolated for Sanger sequencing. We identified the survival variants via Single Nucleotide Polymorphism (SNP) calling on the 
assembled contigs of each DNA pool (Figure 1B \& 1C). The most deviations occur at the nucleotide positions corresponding to amino acid $\mathrm{Arg}^{671}, \mathrm{Asp}^{674}, \mathrm{Gly}^{685}$, and $\mathrm{Val}^{709}$ (Figure 1B \& 1C). These survival variants encode AceCECas9 R671C, D674V, G685A, and V709A/G. The findings were supported by Sanger sequencing of individual colonies, which showed $11 / 20$ to be G685A, 6/20 to be V709A/G, and 3/20 to be D674V. We mapped these amino acid positions onto our previously constructed AceCas9 structure model (Figure S2). Arg ${ }^{671}$, Asp ${ }^{674}$, and $\mathrm{Gly}^{685}$ are located downstream of the helix following the RuvC domain (helix 1) (AceCas9, like other type II-C Cas9s, lacks the second helix following helix 1). Surprisingly, V709 is located outside the predicted hinge region on the $\beta$-strand directly packing against hinge helix 1 (Figure S2), suggesting that structural elements interacting with the hinge helices also impact the allosteric regulation.

In order to confirm the functional activities of the selected AceCECas9, we made individual AceCas9 mutants of G685A and V709A and tested both in-cell and in vitro cleavage assays. Not surprisingly, G685A and V709A had nearly $100 \%$ survival rate in $E$. coli cells harboring the ccdB-plasmid with the 20-nt protospacer (Figure 2A) while the wild-type AceCas9 would not survive (Figures $2 \&$ S1). Consistently, the single-turnover rate of cleavage for both mutants are 3-4-fold of that of the wild-type AceCas9 for either the 24-nt (24mer) or the 20-nt (20mer) protospacer (Figure 2B). Thus, the significantly increased rate of cleavage allows AceCECas9 to effectively eliminate the toxic ccdBplasmid with a 20-nt protospacer, promoting cell survival. 
In order to evaluate if AceCECas9 variants have increased tolerance of DNAsgRNA mismatches, we assessed the survival rates of G685A and V709A in E. coli cells harboring the ccdB-plasmids with mismatched protospacers at position -1 or -4 . These mismatches were previously shown to prevent the wild-type AceCas9 from eliminating the ccdB-plasmid thus leading to death of the E. coli hosts ${ }^{35}$. Similarly, neither G685A nor V709A survived the T(-1)G plasmid (Figure S3), although a small percentage of cells survived for the G(-4)A protospacer, suggesting that AceCECas9 does not significantly escape seed region mismatches in comparison with the wild-type.

In order to identify the molecular basis for the enhanced catalytic efficiency, we performed DNA binding competition experiments to learn if AceCECas9 enhances DNA binding, DNA unwinding, or HNH conformational sampling. To a typical DNA cleavage assay containing $6 \mathrm{nM}$ plasmid target and $250 \mathrm{nM}$ AceCas9 (or AceCECas9), doublestranded DNA oligo competitors were added at increasing concentrations. The fraction of cleavage was then quantified and plotted against the logarithm of competitor concentrations. The plot was then fitted using a standard competition binding model to yield an apparent competitor binding constant, $\mathrm{K}_{\mathrm{I}}$. The wild-type protospacer DNA oligo has nearly identical $\mathrm{K}_{\mathrm{I}}$ for both AceCas9 and AceCECas9 (Figure 3A). The T(-1)G protospacer DNA oligo is a worse competitor than the wild-type but again with equal $\mathrm{K}_{\mathrm{I}}$ for both AceCas9 and AceCECas9, suggesting that AceCECas9 does not enhance, in comparison with AceCas9, DNA binding for either the wild-type or mismatched protospacer (Figure 3A). Finally, AceCas9 and AceCECas9 showed similar binding constants to two bubbled DNA oligos (either complementary or not to sgRNA) (Figure 
3B), indicating that the DNA unwinding was not altered in AceCECas9. These results confirm that the enhanced catalytic efficiency of AceCECas9 is through a process other than DNA binding and unwinding, most likely HNH domain sampling.

Finally, we applied the same directed evolution strategy to the more widely used SpyCas9. Similar to AceCas9, we systematically reduced the base pairs in the protospacer complementary to the guide RNA and determined that 17 base pairs slowed the wildtype SpyCas9 sufficiently to obstruct survival. We then generated a DNA library that encodes SpyCas9 hinge variants (with guide RNA) and transformed it to the cells harboring the $c c d B$ plasmid with a 17-base pair protospacer. Unlike the wild-type SpyCas9 transformation that resulted in no cell survival, the library transformation resulted in significant growth on arabinose containing plates, and after iterative rounds of evolution, a single variant was identified by sequencing (Figure S6). We termed the variant catalytically enhanced SpyCas9 (SpyCECas9). To determine if the SpyCECas9 improves gene editing applications relative to the SpyCas9, we employed both to achieve homology directed repair (HDR)-based insertion of the green fluorescence protein (GFP) gene in the LMNB1 gene within HCT116 cells (Figure 4 \& Figure S7). As shown in Figures 4C, 4D \& 4E, co-transfection of the SpyCECas9-encoding plasmid with the HDR template into HTC116 consistently resulted in 2-fold increase in GFP incorporation versus that of SpyCas9, likely due to its enhanced cleavage of the target DNA.

Our results present the first engineered Cas9s with increased catalytic efficiency through a directed protein evolution method. We demonstrated the effectiveness of this 
method on AceCas9 that yielded enhanced DNA cleavage in bacterial cells. We then used the same strategy to produce SpyCECas9 that doubled the efficiency of the SpyCas9mediated HDR in human colon cancer cells. The catalytically enhanced Cas9s can be applied in areas where they are currently ineffective.

\section{Methods}

\section{Plasmid and Library Construction}

The arabinose-inducible $c c d B$-encoding plasmid (p11-LacY-wtx1) was a gift from Dr. David Edgell (University of Western Ontario). The target sequence was inserted downstream of the $c c d B$ gene using restriction enzymes. Point mutations were made via Q5 site-directed mutagenesis (NEB).

Genes encoding AceCas9 and the sgRNA were inserted into the pACYCDuet vector (Novagen 71147) to form pACYCDuet-Ac9-g119 (20mer) or pACYCDuet-Ac9-g123 (24mer), respectively, as previously described ${ }^{36}$. The hinge-library was created by amplifying the predicted hinge region with Gibson assembly primers via error prone PCR $\left(\mathrm{Ser}^{663}-\mathrm{Gly}^{712}\right)$ at an average rate of 1 mutation per $100 \mathrm{bp}$ and inserting the PCR products into the pACYCDuet-Ac9-g119 vector. The resulting DNA was transformed into DH5 $\alpha$ cells. About 60,000 colonies were obtained after overnight growth on media containing chloramphenicol. The colonies were pooled, grown for 2 hours, then collected for extraction of plasmid DNA.

\section{In vivo selection}


The survival assay was carried out as previously described ${ }^{34-36}$. Briefly, electrocompetent E. coli BW25141 containing the modified p11-LacY-wtx1 (ccdB encoding) plasmid were transformed with 100-200 ng of AceCas9 library plasmids. Cells were then recovered in prewarmed Super Optimal Broth (SOB) for 30 minutes with shaking at $37{ }^{\circ} \mathrm{C}$ before $0.05 \mathrm{mM}$ IPTG was added, and recovery continued for an additional 60 minutes. Transformants were then plated on LB agar plates containing either chloramphenicol or chloramphenicol and $10 \mathrm{mM}$ arabinose and incubated at $37^{\circ} \mathrm{C}$ for 16 20 hours. Colonies were counted manually, and survival rates were calculated by dividing the colony forming units (CFU) of arabinose-containing plates by that of the chloramphenicol only plates.

\section{Next Generation Sequencing}

The hinge region of each survival pool was PCR amplified with primers containing Illumina indexes. Adaptors were added to these PCR products and then the pooled libraries were subjected to 300 cycle single-end sequencing using MiSEQ (Illumina). Data was analyzed using Geneious 9.0.5 NGS analysis tools.

\section{Protein Expression and Purification}

AceCas 9 and mutant variants were expressed and purified as previously described $^{36}$. Briefly, harvested cells in a lysis buffer were lysed via sonication. Clarified lysate was loaded onto an Ni-NTA agarose (Qiagen) column. Elutant was further purified via a HiTrap Heparin-HP (GE Healthcare) column and then a size exclusion column 
(HiLoad 26/60 Superdex 200 increase GE Healthcare) before concentration and storage at $-80^{\circ} \mathrm{C}$.

\section{In-vitro transcription and Purification of sgRNA}

DNA oligos coding for a T7 promotor followed by the sgRNA were annealed in 10x PCR buffer by heating in boiling water for 5 minutes followed by slow cooling. Invitro transcription was performed by adding $1 \mathrm{uM}$ of this dsDNA to $50 \mathrm{mM}$ Tris $\mathrm{pH} 8.0$, $40 \mathrm{mM} \mathrm{MgCl}_{2}, 10 \mathrm{mM}$ dithiothreitol (DTT), 2 mM Spermindine, $5 \mathrm{mM}$ ATP, UTP, CTP, and GTP pH 8.0, 0.1\% Triton X-100, and $0.15 \mathrm{mg} / \mathrm{mL}$ of homemade T7 RNA Polymerase, and incubating at $37^{\circ} \mathrm{C}$ overnight. The reaction was quenched the following morning by adding $50 \mathrm{mM}$ EDTA. The sgRNA was then extracted via phenol/chloroform $\mathrm{pH} 4.5$ extraction and purified via 10\% denaturing PAGE.

\section{Plasmid Cleavage Assay}

Plasmid cleavage assays were performed as previously described ${ }^{34-36}$. Briefly, 500 nM pre-annealed AceCas9: sgRNA complex was incubated with $6 \mathrm{nM}$ plasmid DNA in cleavage buffer $(20 \mathrm{mM}$ Tris $\mathrm{pH} 7.5,150 \mathrm{mM} \mathrm{KCl,} 2 \mathrm{mM}$ DTT, $10 \mathrm{mM} \mathrm{MgCl} 2,5 \%$ glycerol) at $50{ }^{\circ} \mathrm{C}$ for a given time. Reactions were then stopped by adding $5 \mathrm{x}$ stop buffer (25 mM Tris $\mathrm{pH}$ 7.5, $250 \mathrm{mM}$ EDTA, 1\% SDS, 0.05\% w/v bromophenol blue, 30\% glycerol) and resolved on a 1x TBE $0.5 \%$ agarose gel containing Ethidium bromide. Gels were imaged by the ChemiDoc XRS System (Bio-Rad).

\section{Single Turnover Kinetics}


Plasmid kinetics assays were performed in a similar manner as the plasmid cleavage assays, but all reagents (DNA, RNP complex, and Eppendorf tubes) were kept ice cold before reactions were initiated. After initiation of reaction, samples were taken from $50{ }^{\circ} \mathrm{C}$ water bath in triplicate at each time point and placed on ice, then ice cold stop buffer was quickly added. Gels were analyzed by Image Lab Version 5.2.1 build 11 (Bio-Rad) and fitted to an exponential plateau curve in Prism 8.1.1 to extract $\mathrm{k}_{\text {cleavage. }}$

\section{DNA Binding Competition Assays}

Oligonucleotides were annealed by heating in $95{ }^{\circ} \mathrm{C}$ water bath followed by slow cooling. Serial dilutions of oligos were made from $25 \mathrm{uM}$ to $0.063 \mathrm{uM}$, and added to $6 \mathrm{nM}$ plasmid DNA. Plasmid/oligo mixture was then added to annealed RNP complex (250 nM AceCas9 (or AceCECas9) and $250 \mathrm{nM}$ sgRNA) and allowed to react for 15 minutes at 50 ${ }^{\circ} \mathrm{C}$. Reactions were stopped with stop buffer, resolved on agarose gels before being visualized, and quantified as before.

\section{CRISPR-mediated Homology Directed Repair (HDR) Assay in HCT116 cells}

HCT116 cells were maintained in McCoy's 5A media supplemented with $10 \%$ fetal bovine serum and $1 \%$ penicillin/streptomycin at $37{ }^{\circ} \mathrm{C}$ with $5 \% \mathrm{CO}_{2}$. The plasmids encoding the wild-type SpyCas9 (px330, Addgene \#42230) or its catalytically enhanced variant, SpyCECas9 (modified px330), targeting the LMNB1 gene (GGGGTCGCAGTCGCCATGGC) (2 ug) and an LMNB1-mEGFP containing repair vector (Addgene plasmid \#87422) (3 $\mu \mathrm{g}$ ) were nucelofected into HCT116 cells grown to $70-80 \%$ confluency using the Lonza SE cell line 4-D Nucleofector solution kit with pulse 
code EN113. 48-hours post-nucleofection, cells were evaluated for the presence of GFP using FACSCanto.

\section{References}

1. Kleinstiver, B.P. et al. High-fidelity CRISPR-Cas9 nucleases with no detectable genome-wide off-target effects. Nature 529, 490-495 (2016).

2. Rees, H.A. et al. Improving the DNA specificity and applicability of base editing through protein engineering and protein delivery. Nat Commun $\mathbf{8}$, 15790 (2017).

3. Slaymaker, I.M. et al. Rationally engineered Cas9 nucleases with improved specificity. Science 351, 84-88 (2016).

4. Chen, J.S. et al. Enhanced proofreading governs CRISPR-Cas9 targeting accuracy. Nature 550, 407-410 (2017).

5. Gaudelli, N.M. et al. Programmable base editing of $\mathrm{A}^{*} \mathrm{~T}$ to $\mathrm{G}^{*} \mathrm{C}$ in genomic DNA without DNA cleavage. Nature 551, 464-471 (2017).

6. Guilinger, J.P., Thompson, D.B. \& Liu, D.R. Fusion of catalytically inactive Cas9 to FokI nuclease improves the specificity of genome modification. Nat Biotechnol 32, 577-582 (2014).

7. Hu, J.H. et al. Evolved Cas9 variants with broad PAM compatibility and high DNA specificity. Nature 556, 57-63 (2018).

8. Li, C. et al. Expanded base editing in rice and wheat using a Cas9-adenosine deaminase fusion. Genome Biol 19, 59 (2018).

9. Lee, J.K. et al. Directed evolution of CRISPR-Cas9 to increase its specificity. Nat Commun 9, 3048 (2018).

10. Didovyk, A., Borek, B., Tsimring, L. \& Hasty, J. Transcriptional regulation with CRISPR-Cas9: principles, advances, and applications. Curr Opin Biotechnol 40, 177-184 (2016).

11. Doench, J.G. et al. Optimized sgRNA design to maximize activity and minimize off-target effects of CRISPR-Cas9. Nat Biotechnol 34, 184-191 (2016).

12. Doench, J.G. et al. Rational design of highly active sgRNAs for CRISPR-Cas9mediated gene inactivation. Nat Biotechnol 32, 1262-1267 (2014).

13. Graf, R., Li, X., Chu, V.T. \& Rajewsky, K. sgRNA Sequence Motifs Blocking Efficient CRISPR/Cas9-Mediated Gene Editing. Cell Rep 26, 1098-1103 e1093 (2019).

14. Gagnon, J.A. et al. Efficient mutagenesis by Cas9 protein-mediated oligonucleotide insertion and large-scale assessment of single-guide RNAs. PLoS One 9, e98186 (2014).

15. Lee, C.M., Cradick, T.J. \& Bao, G. The Neisseria meningitidis CRISPR-Cas9 System Enables Specific Genome Editing in Mammalian Cells. Mol Ther 24, 645-654 (2016). 
16. Harrington, L.a.P.-E.D.a.C.J.S.a.M.E.a.S.B.T.a.K.N.C.a.D.J.A.a.Z.F.a. A thermostable Cas9 with increased lifetime in human plasma. Doi.Org 60, 242-255 (2015).

17. Ma, E., Harrington, L.B., O'Connell, M.R., Zhou, K. \& Doudna, J.A. SingleStranded DNA Cleavage by Divergent CRISPR-Cas9 Enzymes. Mol Cell 60, 398-407 (2015).

18. Mir, A., Edraki, A., Lee, J. \& Sontheimer, E.J. Type II-C CRISPR-Cas9 Biology, Mechanism, and Application. ACS Chem Biol 13, 357-365 (2018).

19. Mushtaq, M. et al. Harnessing Genome Editing Techniques to Engineer Disease Resistance in Plants. Front Plant Sci 10, 550 (2019).

20. Jaganathan, D., Ramasamy, K., Sellamuthu, G., Jayabalan, S. \& Venkataraman, G. CRISPR for Crop Improvement: An Update Review. Front Plant Sci 9, 985 (2018).

21. Jiang, F. \& Doudna, J.A. CRISPR-Cas9 Structures and Mechanisms. Annu Rev Biophys 46, 505-529 (2017).

22. Sternberg, S.H., Redding, S., Jinek, M., Greene, E.C. \& Doudna, J.A. DNA interrogation by the CRISPR RNA-guided endonuclease Cas9. Nature 507, 6267 (2014).

23. Sorek, R., Lawrence, C.M. \& Wiedenheft, B. CRISPR-mediated adaptive immune systems in bacteria and archaea. Annu Rev Biochem 82, 237-266 (2013).

24. Singh, D., Sternberg, S.H., Fei, J.Y., Doudna, J.A. \& Ha, T. Real-Time Observation of DNA Recognition by the RNA-Guided Endonuclease Cas9 using SingleMolecule FRET. Biophys J 108, 26a-26a (2015).

25. Raper, A.T., Stephenson, A.A. \& Suo, Z. Functional Insights Revealed by the Kinetic Mechanism of CRISPR/Cas9. J Am Chem Soc 140, 2971-2984 (2018).

26. Gong, S.Z., Yu, H.H., Johnson, K.A. \& Taylor, D.W. DNA Unwinding is the Primary Determinant of CRISPR-Cas9 Specificity. Biophys J 114, 251a-251a (2018).

27. Sternberg, S.H., LaFrance, B., Kaplan, M. \& Doudna, J.A. Conformational control of DNA target cleavage by CRISPR-Cas9. Nature 527, 110-113 (2015).

28. Jiang, F. et al. Structures of a CRISPR-Cas9 R-loop complex primed for DNA cleavage. Science 351, 867-871 (2016).

29. Palermo, G. et al. Key role of the REC lobe during CRISPR-Cas9 activation by 'sensing', 'regulating', and 'locking' the catalytic HNH domain. Q Rev Biophys 51 (2018).

30. Jackson, R.N., van Erp, P.B., Sternberg, S.H. \& Wiedenheft, B. Conformational regulation of CRISPR-associated nucleases. Curr Opin Microbiol 37, 110-119 (2017).

31. Zuo, Z. \& Liu, J. Structure and Dynamics of Cas9 HNH Domain Catalytic State. Sci Rep 7, 17271 (2017).

32. Palermo, G. et al. Protospacer Adjacent Motif-Induced Allostery Activates CRISPR-Cas9. J Am Chem Soc 139, 16028-16031 (2017).

33. Zeng, Y. et al. The initiation, propagation and dynamics of CRISPR-SpyCas9 Rloop complex. Nucleic Acids Res 46, 350-361 (2018). 
34. Hand, T.H., Das, A. \& Li, H. Directed evolution studies of a thermophilic Type II-C Cas9. Methods Enzymol 616, 265-288 (2019).

35. Hand, T.H. et al. Phosphate Lock Residues of Acidothermus cellulolyticus Cas9 Are Critical to Its Substrate Specificity. ACS Synth Biol (2018).

36. Tsui, T.K.M., Hand, T.H., Duboy, E.C. \& Li, H. The Impact of DNA Topology and Guide Length on Target Selection by a Cytosine-Specific Cas9. ACS Synth Biol 6, 1103-1113 (2017).

\section{Acknowledgments}

We thank D. Edgell for providing cells and plasmids for in vivo study; B. Washburn, C. Pye, and Kristina Poduch of the FSU Molecular Cloning Facility for the cloning experiments and discussion; S. Miller and A. Brown of the FSU Sequencing facility for assistance with NGS library preparation and sequencing. This work was supported by NIH grants R01 GM099604 to H.L. and R01 GM083337 to D.M.G.

\section{Author Contributions}

T.H. and H.L. designed all experiments. T.H. designed primers for $c c d B$ plasmid variants and AceCas9 plasmid libraries, created AceCas9 plasmid libraries, prepared DNA for NGS and analyzed results, purified AceCas9 and variants obtained from library screens, and performed in vitro DNA cleavage assays. M.R., C.S., and E.S. performed in vivo survival assays with AceCas9 plasmid libraries for iterative rounds, prepped DNA for each pool, and analyzed Sanger sequencing results. M.R. and K.K. transfected HCT116 cells and analyzed results. E.S. designed primers for variants obtained from library screens. T.H. and H.L analyzed data, wrote and edited manuscript and figures.

\section{Conflict of interest}

The authors declare that they have no conflict of interest. 


\section{Figure Legends}

Figure 1: Selection of catalytically enhanced AceCas9. A. Top, Domain organization of AceCas9 with amplified view of linker II (L-II) with primers used for error-prone PCR primers. Bottom, Directed protein evolution selection strategy. pACAY-AceCas9-g123 and pACAY-AceCas9-g119 co-express AceCas9 and the single guide RNA with 24mer and 20mer guide, respectively. B. Sequence logo of consensus sequence from NGS analysis of final pool. The height of each letter is proportional to the observed frequency of each nucleotide in the alignment column. The locations with highest deviation are marked by their corresponding amino acid positions. C. Pie charts illustrate enrichment of amino acids at the four highest frequency sites. Pool 0 reflects enrichment values in the starting library whereas pool 5 reflects those from the last round of survival colonies. Single letter for amino acids are used.

Figure 2: Verification of the catalytically enhanced AceCas9 by survival assay and kinetic analysis. A, Plasmids encoding the selected AceCas9 mutants were transformed into ccdB harboring cells that are plated on plates with (Ara+) or without arabinose (Ara-) respectively. Images of the plates are shown with the corresponding survival rate plotted below. B, Single-turnover DNA-cleavage experiments and the resulting rates of the AceCECas9 with either the 20 mer spacer or the 24 mer spacer sgRNA. Molar excess of AceCas9-sgRNA complex were incubated with plasmid DNA for various times at $50{ }^{\circ} \mathrm{C}$ before being resolved and visualized on agarose gels. Kinetic experiments were performed in triplicate. 
Figure 3: DNA binding competition assay results. The DNA oligo substrates used are schematically shown as wild-type (WT), T(-1)G/A(+1)C (dsDNA with mismatch to sgRNA at position +1$), \mathrm{T}(-1) \mathrm{T} / \mathrm{A}(+1) \mathrm{C}$ (bulged DNA oligo at position +1 that mismatches with sgRNA), and non-target strand bulge (bulged DNA oligo at position at +1 that matches sgRNA). For each competition binding experiment, the cleavage gel image and fraction of cleavage versus competitor concentration at logarithmic scale are shown. Solid curves are fitted theoretical curves and fitted $\mathrm{K}_{\mathrm{I}}$ values are indicated. A, Competition binding result for the wild-type or $\mathrm{T}(-1) \mathrm{G}$ oligo with AceCas9 (WT) and the V709A variant. B, Competition binding result for the bulged oligos with AceCas9 (WT) and the V709A variant.

Figure 4: Gene insertion facilitated by the wild-type and the catalytically enhanced SpyCas9 in HCT116 cells. A, Cartoon illustration depicting the genome insertion process. The px330 vector co-expressing either the wild-type SpyCas9 or the SpyCECas9 with the guide sgRNA targeting the LMNB1 gene is co-transfected with the repair vector containing mEGFP flanked by $1 \mathrm{~kb}$ homology arms (HAs). Each transfection is performed with the Lonza SE cell line 4-D Nucleofector solution kit by electroporating one million HCT116 cells in an electro-cuvette. Cells are plated and cultured for 48-hours post transfection (positive cells are depicted as grey with a green ring around the nuclear lamina). The HCT116 cells that successfully achieved homology directed repair (HDR) by incorporation of the green fluorescent protein (GFP) are detected, sorted, collected via flow cytometry and expanded for imaging. B, Detailed scheme of the CRISPR-Cas9-mediated green 
fluorescence protein (GFP) tagging strategy. 1) The crRNA (purple) co-expressing with the wild-type SpyCas9 (px330, Addgene \#42230) or the catalytically enhanced (CE) SpyCECas9 on a modified px330 vector targets a site upstream of exon 1 (red) of the LMNB1 gene. 2) A repair vector containing 1kb homology arms (HAs) flanking the GFPencoding sequence fused with a linker sequence (AICSDP-10:LMNB1-mEGFP, Addgene \#87422). 3) Successfully edited target locus following SpyCas9 cleavage and homology directed repair contains an in-frame insertion of the GFP after the start codon of LMNB1 exon 1. C, The observed GFP signal of the wild-type and the catalytically enhanced SpyCas9. Flow cytometry plots displaying GFP fluorescence intensity (x-axis) versus RFP fluorescence intensity (y-axis; to control for auto-fluorscence) following 48-hour posttransfection for the wild-type (SpyCas9) and catalytically enhanced (SpyCECas9). GFP positive cells fall within the $\mathrm{P} 2$ gate. The negative control is obtained from cells transfected with the repair vector and SpyCas9 without a sgRNA. Experiments were conducted in triplicate (Figure S7). D, Bar graph displaying the averages of the quadruplicate measurement for both SpyCas9 and SpyCECas9 and error bars displaying standard deviation. Statistical significance was determined by an unpaired t-test $\left({ }^{*} p \leq 0.05\right.$, exact value shown below the asterisk). E, Representative fluorescence microscopy images of GFP-tagged LMNB1 in HCT116 cells obtained on a DeltaVision (GE Life Sciences) microscope. The cell nuclei were stained with DAPI. 


\section{AceCas9}

\begin{tabular}{|l|l|l|l|l|l|l|l|l|l|}
\hline 1 & \multicolumn{1}{c|}{479} & 510 & 525 & 669 & 705 & 832 & 1138 \\
\hline RuvC-I & REC & RuvC-II & L-I & HNH & L-II & RuvC-III & PID \\
\hline
\end{tabular}

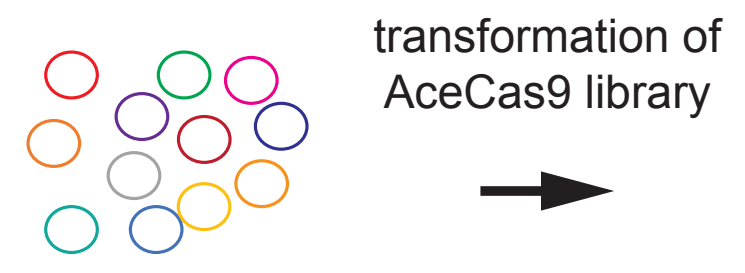

pACYCDuet-AceCas9-g123 (chloramphenicol')
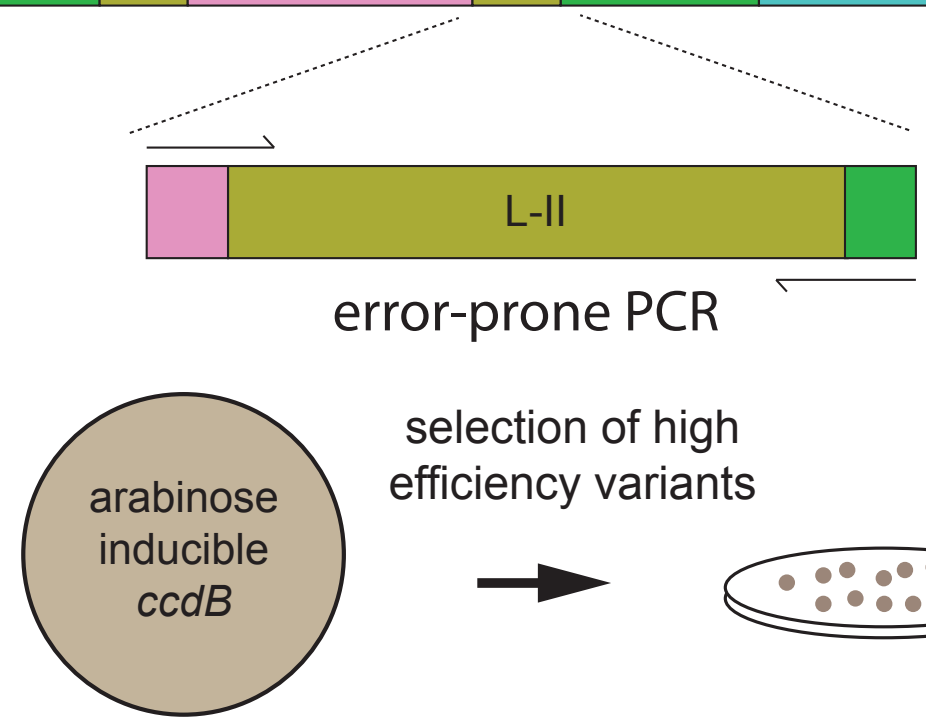

BW25141(DE3) E. coli selection of high efficiency variants
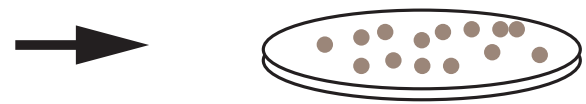

+arabinose

+chloramphenicol

\section{R671 D674}

G685

V709

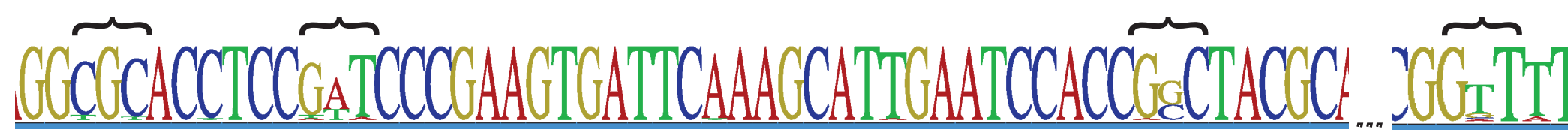

R671

other amino acids

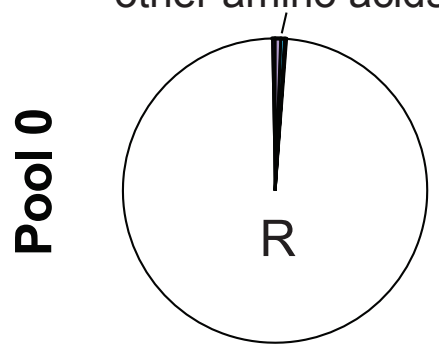

$\frac{10}{8}$

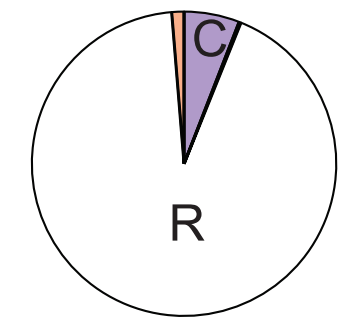

D674

other amino acids
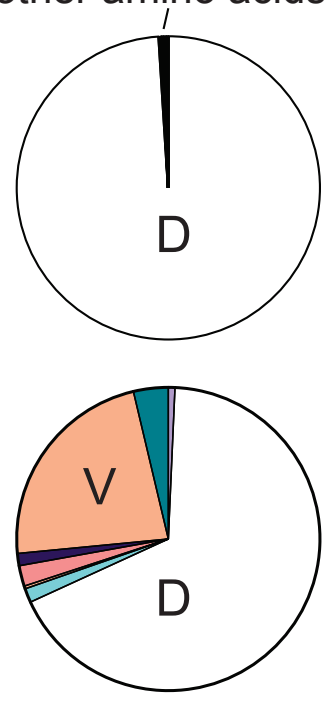

G685

other amino acids
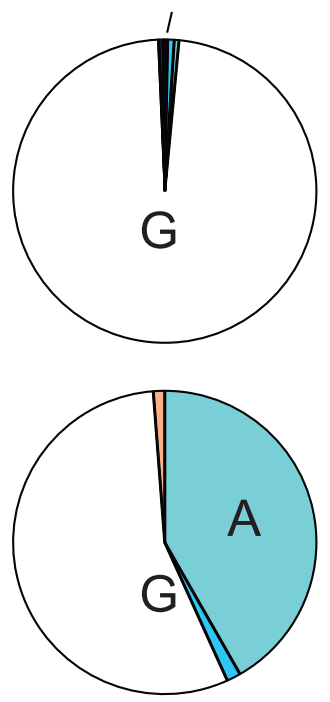

V709

other amino acids
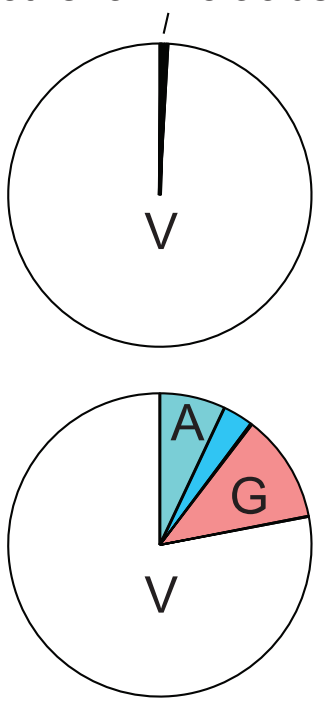
A

ccdB Survival G685A

V709A
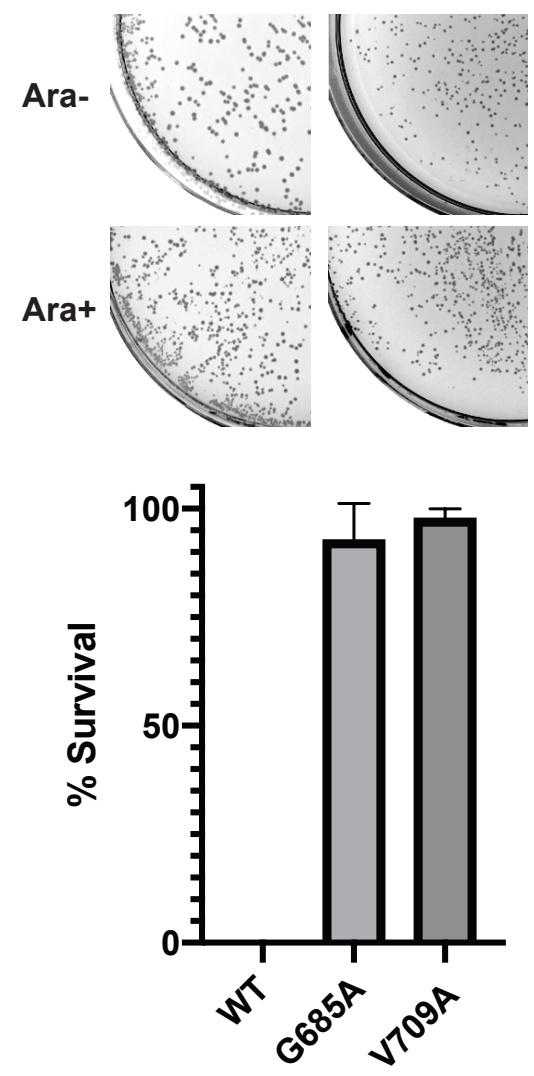

G685A-20mer

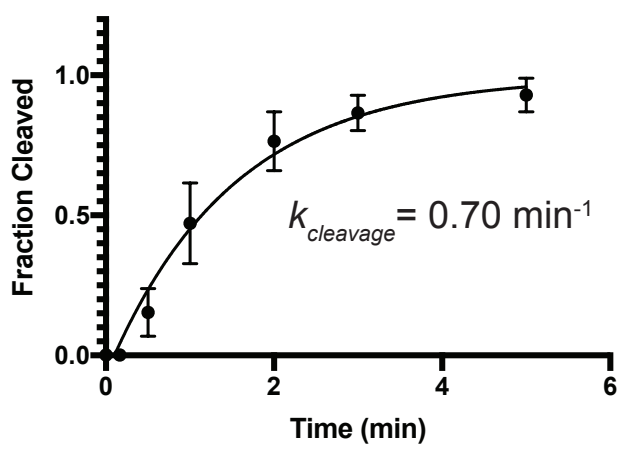

G685A- 24mer

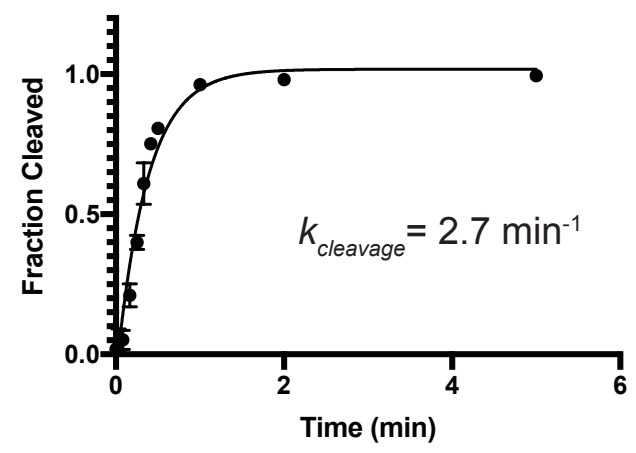

V709A-20mer

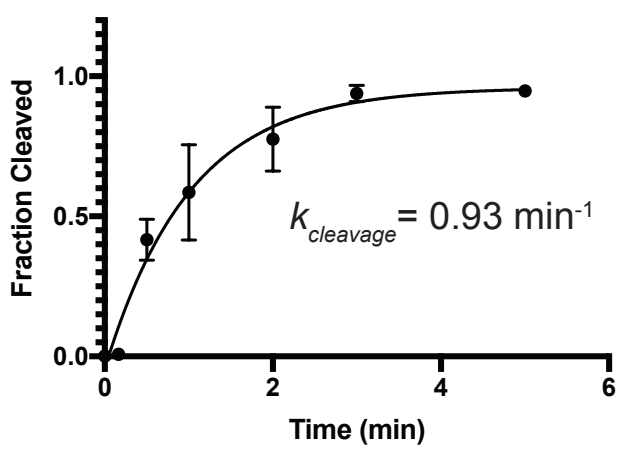

V709A- 24mer

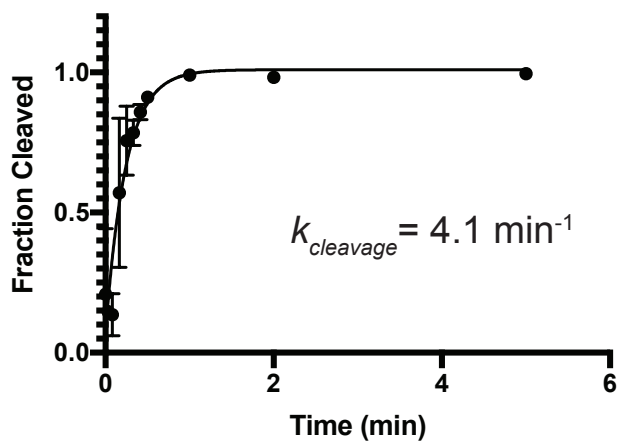




\section{WT Competitor}

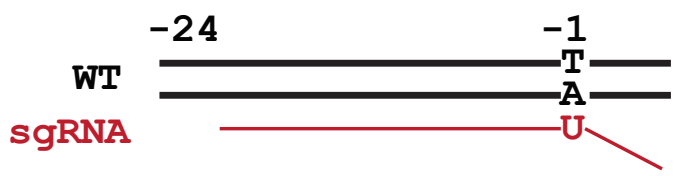

AceCas9

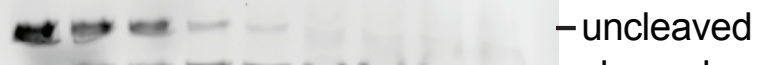

AceCECas9
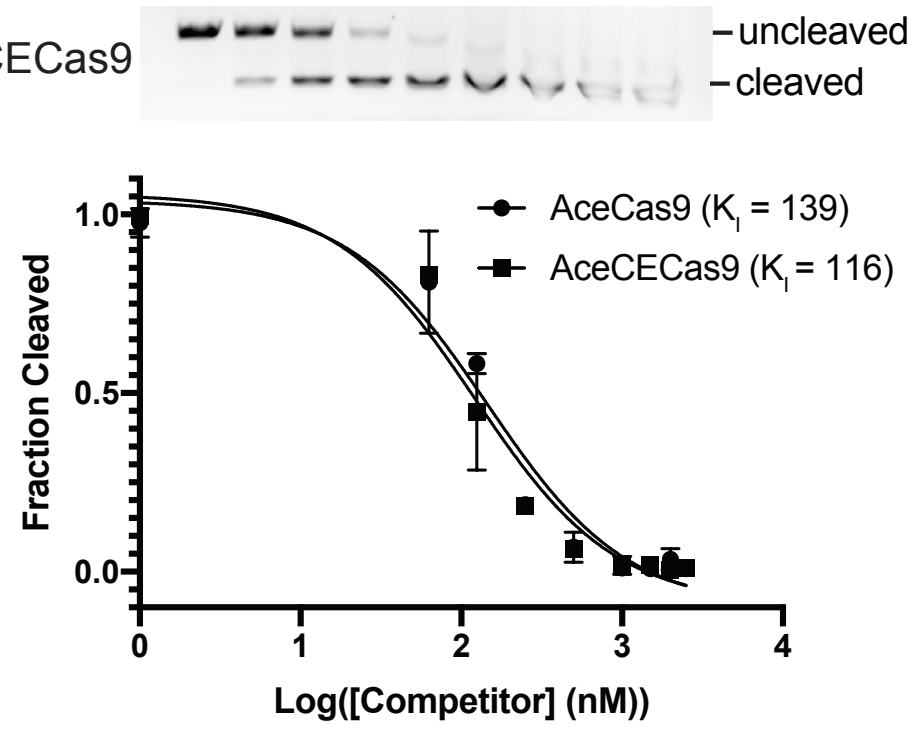

C

\section{Bulged Competitor}

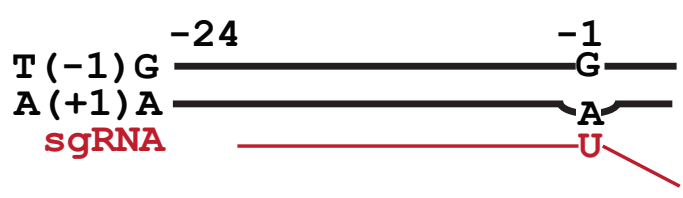

AceCas9 AceCECas9
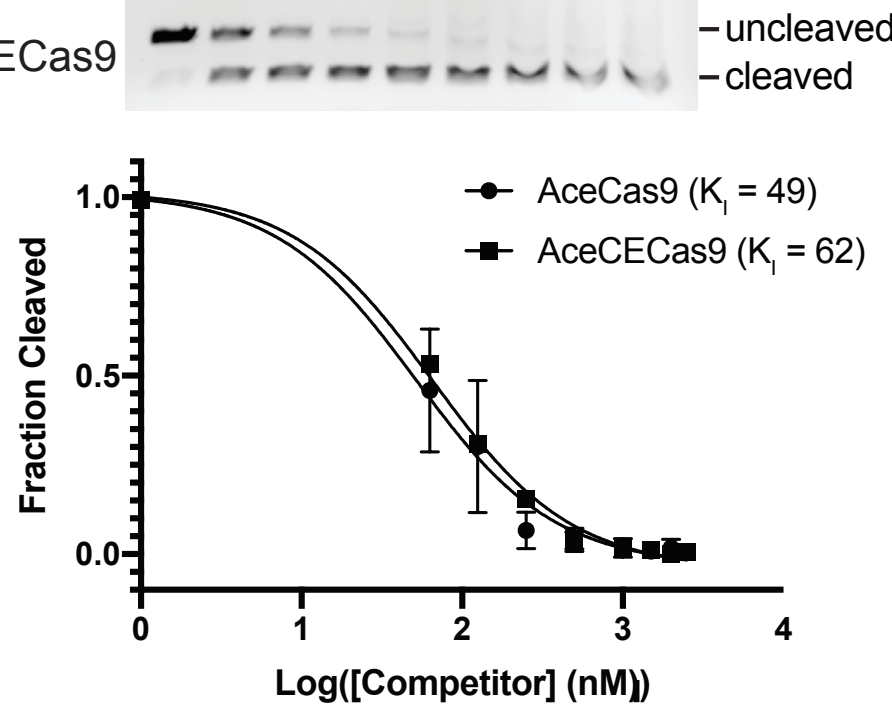

\section{Mismatch Competitor}

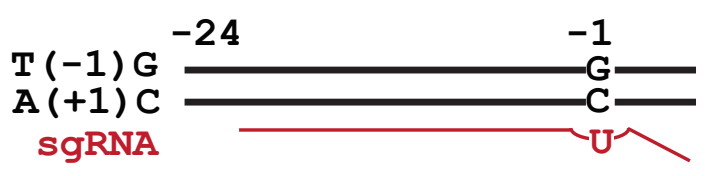

AceCas9

- uncleaved

-cleaved

AceCECas9

- uncleaved

- cleaved

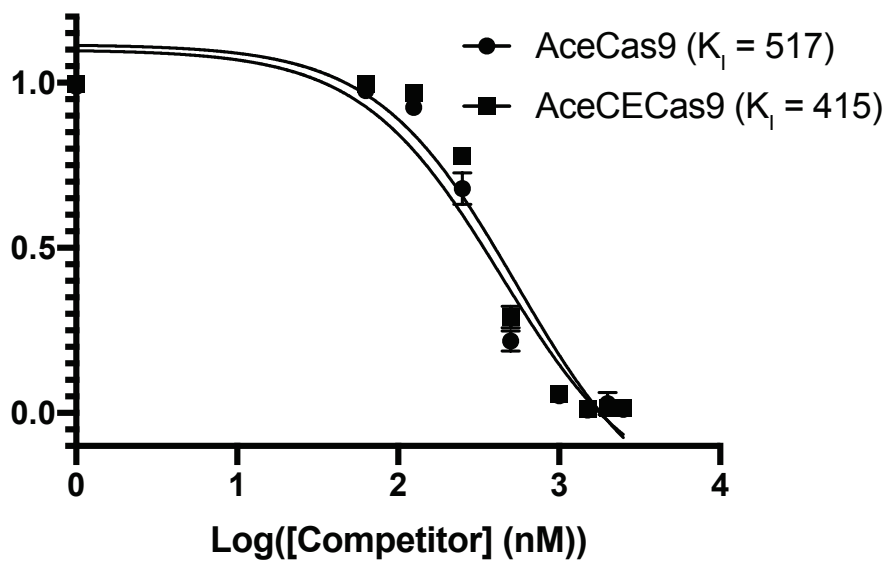

D Bulged \& Mismatch Competitor
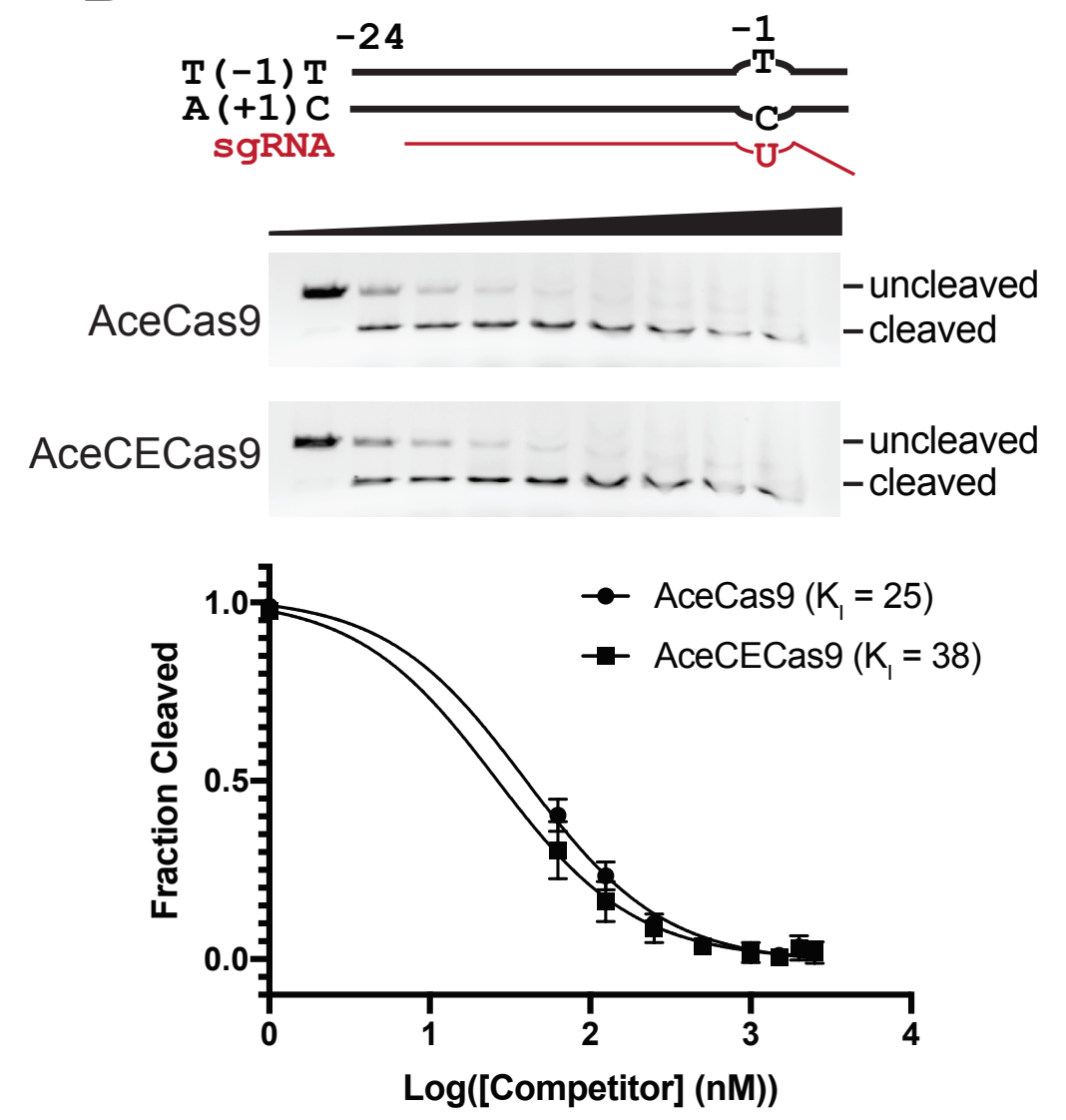
A bioRxiv preprint doi: https://doi.org/10.1101/2020.07.01.183194; this version posted July 1, 2020. The copyright holder for this preprint (which 14 istion

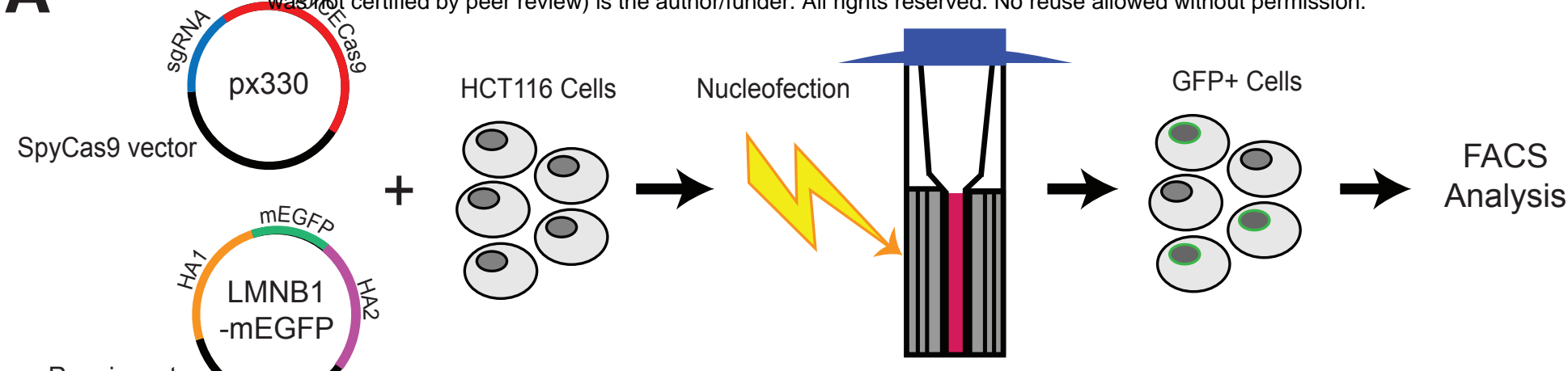

B

SpyCas9 encoded by px330

1) Target Locus (LMNB1)

(4) CrRNA

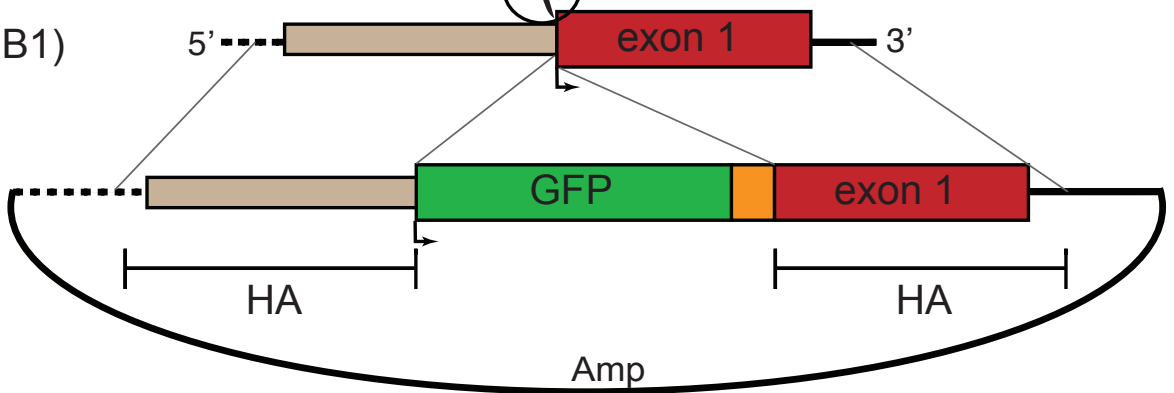

3) HDR Edited LMNB1
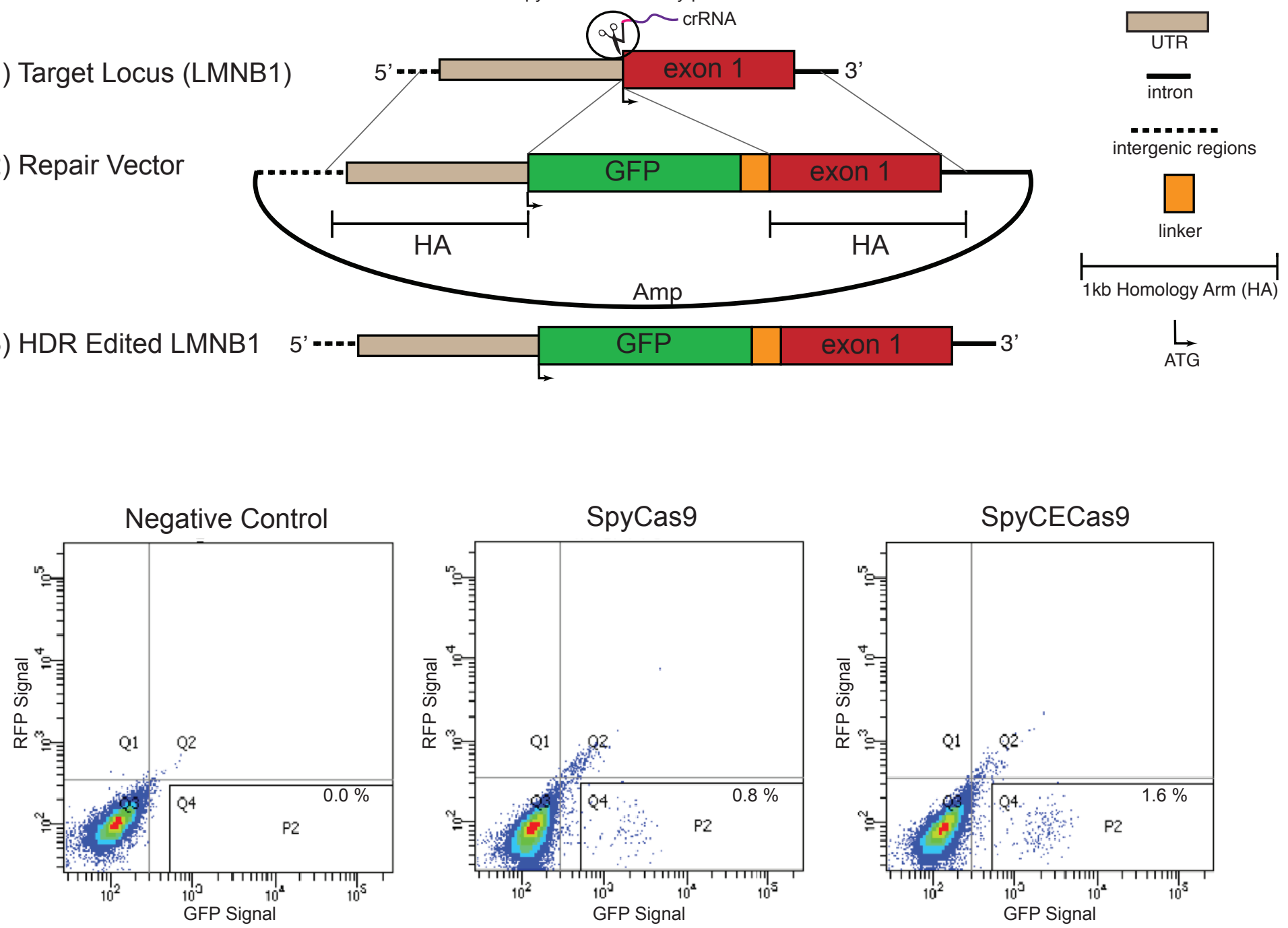

D

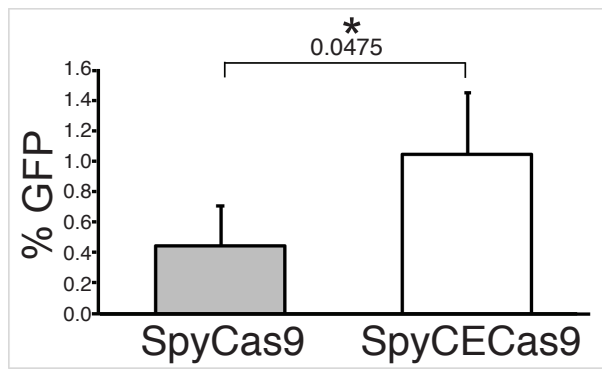

DAPI

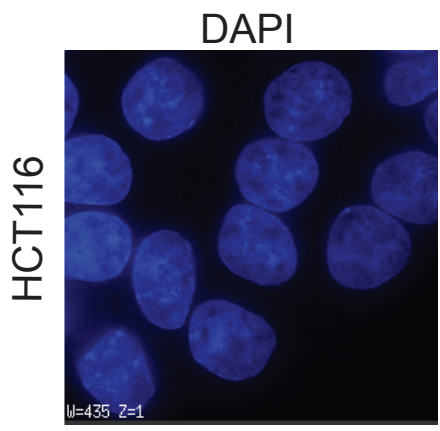

mEGFP LMNB1

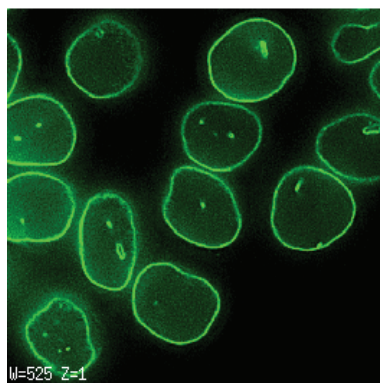

Merge

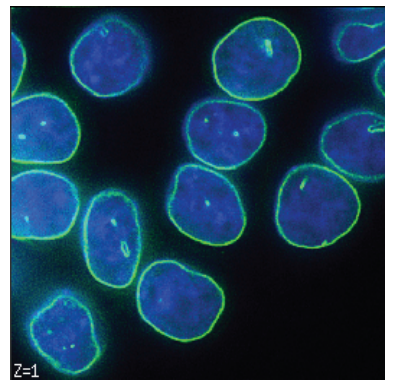


bioRxiv preprint doi: https://doi.org/10.1101/202B07.01.183194; this version posted July 1, 2920. The copyright holder for this preprint (which

$\triangle$ was not certified by peer review) is 4 e author/funder. All rights reserved. Noreluse allowed without permission.
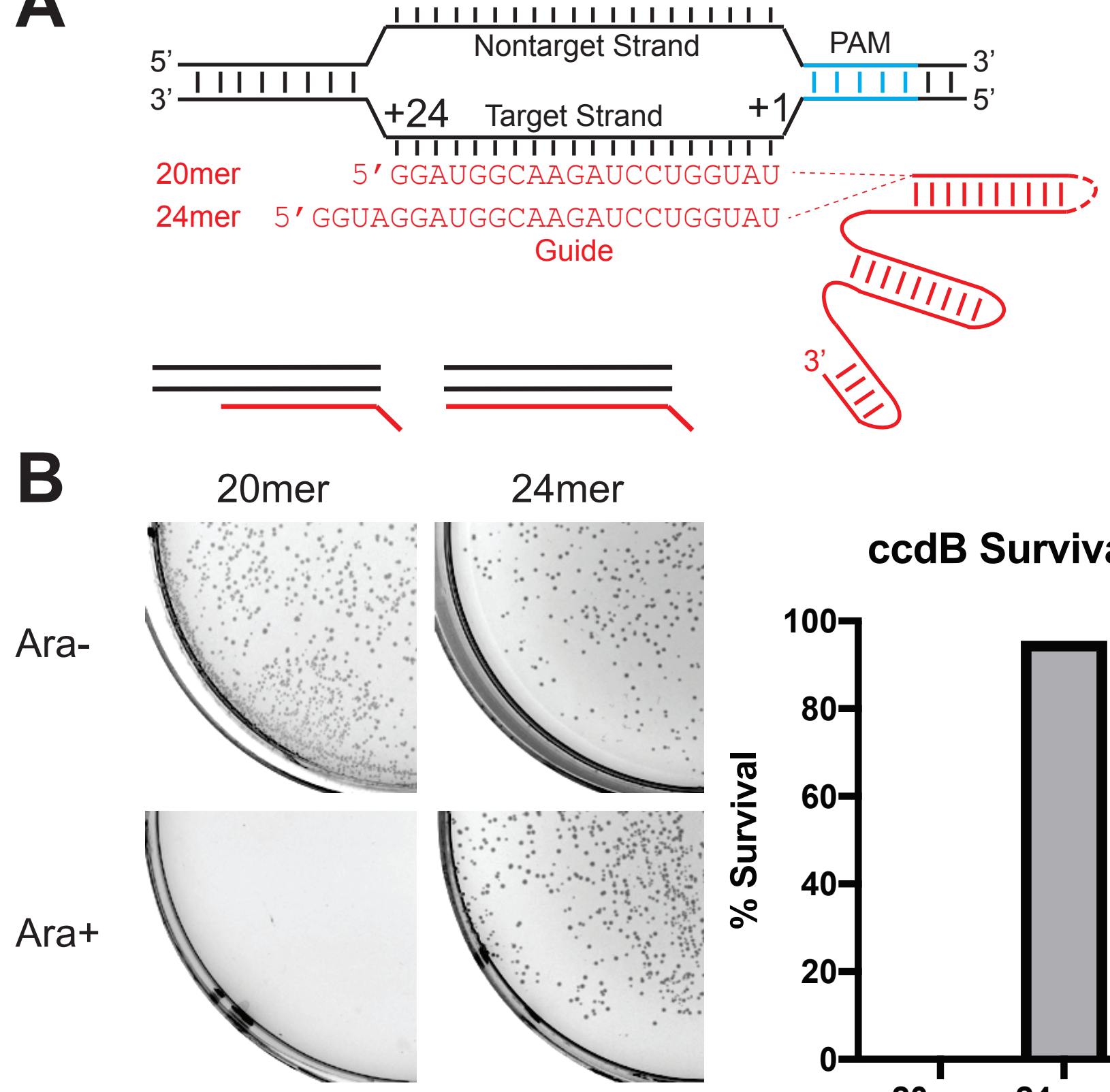

ccdB Survival

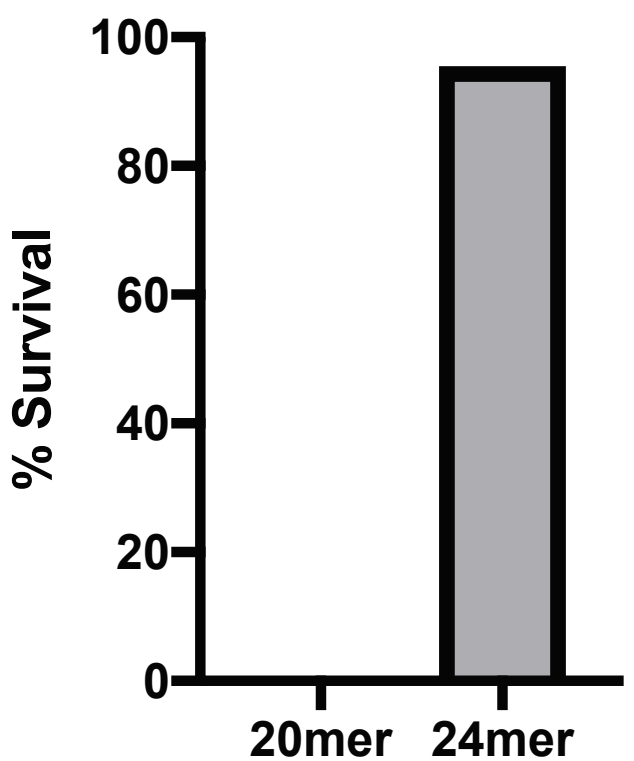

Supplemental Figure 1: Sensitivity of AceCas9 to spacer length. A, Structural features of target DNA and sgRNA used in this study. The numbering of the terminal protospacer nucleotides are indicated. B, Plasmids encoding AceCas9 with either 20mer (pACAY-AceCas9-g119) or 24mer (pACAY-AceCas9-g123) guide RNA were transformed into ccdB harboring cells. Images of plates in the absence (Ara-) and the presence of arobinose (Ara+) are shown with caculated survival rates plotted as bar diagrams to the right. 


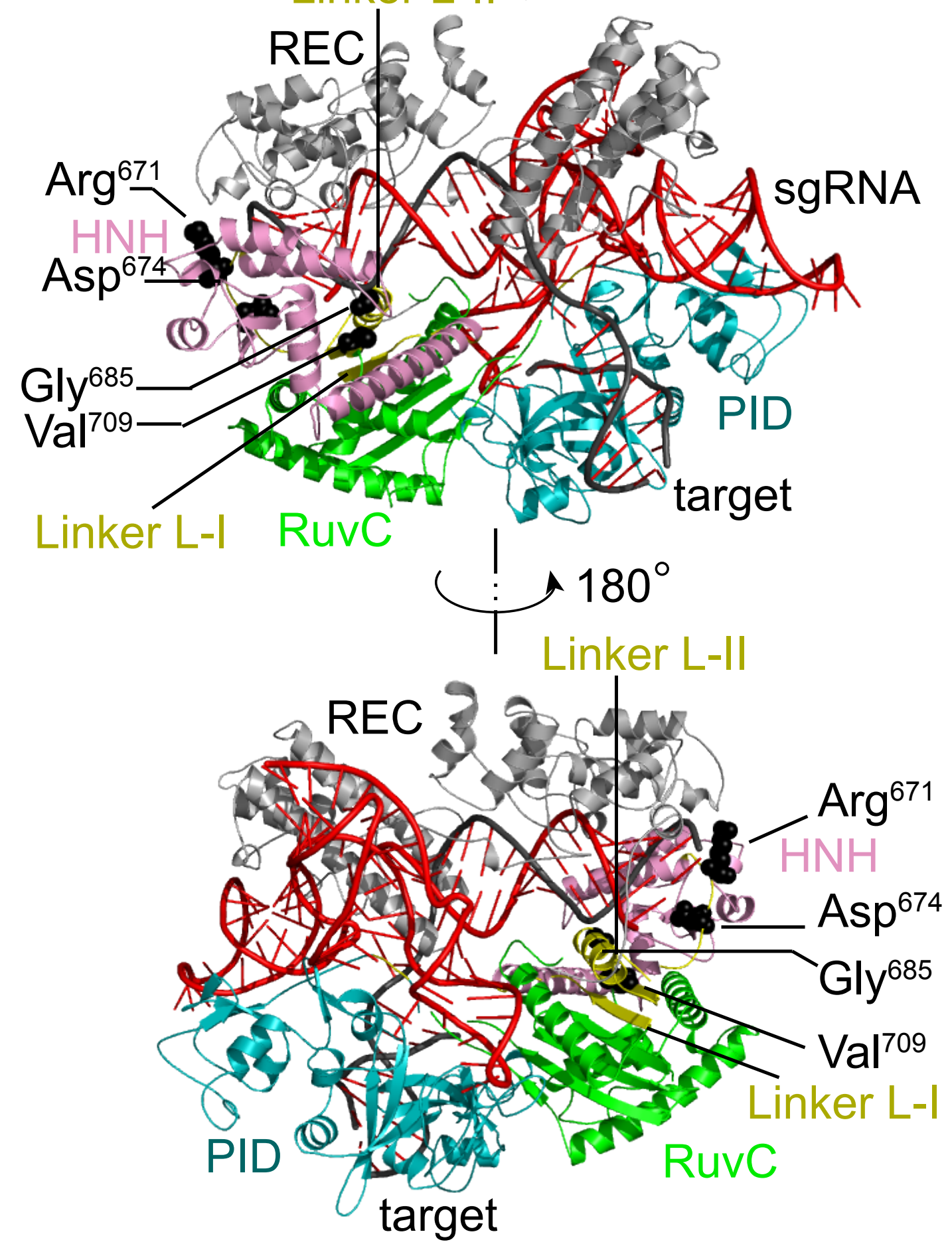

Supplemental Figure 2: Mapping catalytically enhanced mutations on a modeled AceCas9 structure. AceCas9 domains are colored and labeled. Target DNA is shown in black and sgRNA shown in red. The mutations are shown as black spheres as labeled. $\mathrm{REC}$, nucleic acid recognition domain (gray); $\mathrm{HNH}$, $\mathrm{HNH}$ catalytic domain (pink); RuvC, RuvC catalytic domain (green); PID, PAM interacting domain (teal), and Linkers L-I and L-II (yellow). 
B
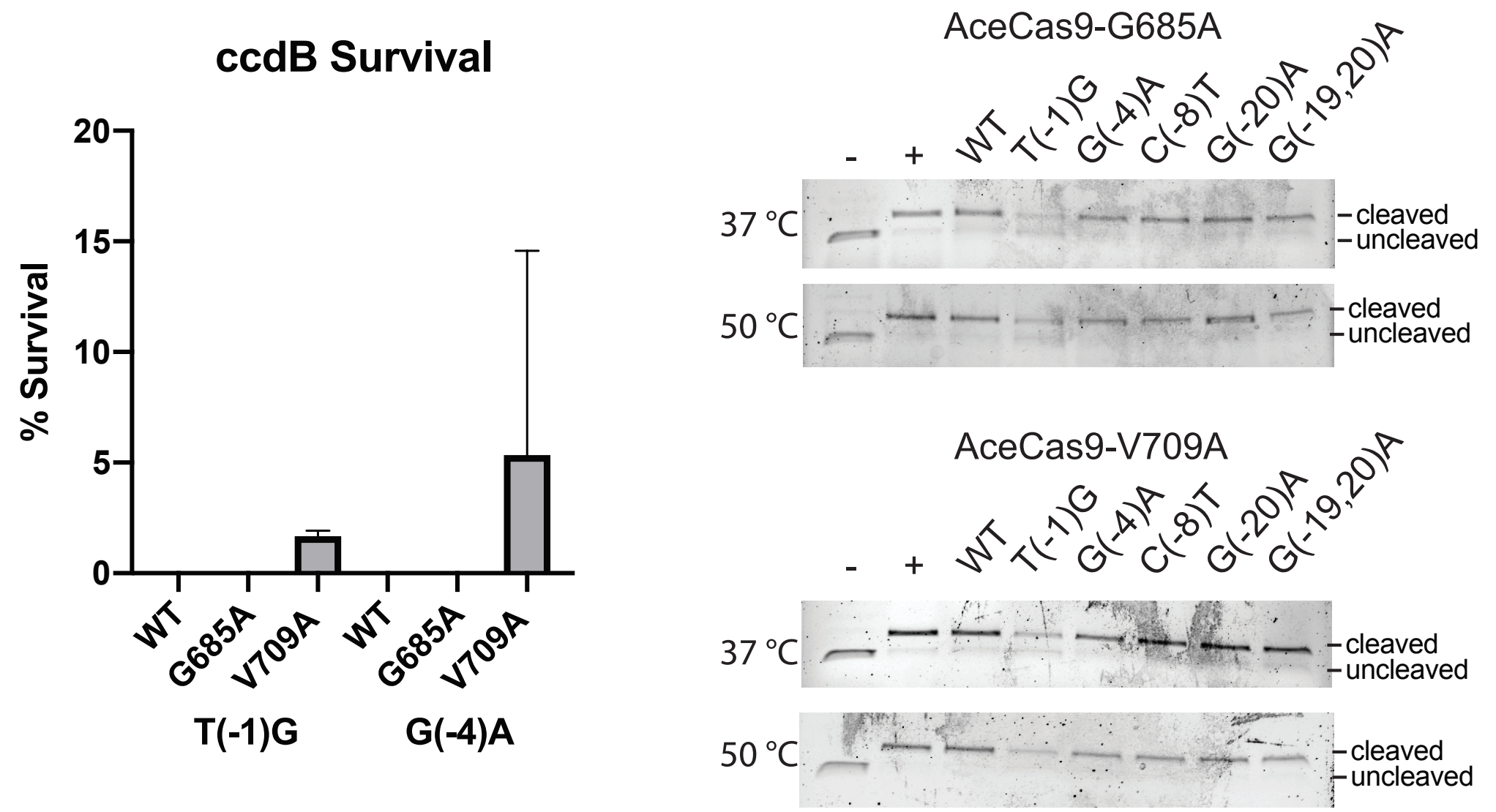

Supplemental figure 3: Off-target analysis of AceCECas9. A, Plasmids encoding AceCas9 (WT or AceCECas9 variants) were transformed into ccdB T(-1)G or G(-4)A harboring cells. Caculated survival rates shown in a bar graph. Error bars represent standard deviation (S.D.) from triplicates. B, in vitro cleavage reactions with AceCECas9 variants with plasmid DNA harboring mismatches in the protospacer. Molar excess of AceCECas9 was incubated with plasmids for 60 minutes at $50^{\circ} \mathrm{C}$ or $37^{\circ} \mathrm{C}$ before being separated and visualized on agarose gels. 
V709A-20mer guide

time (min)

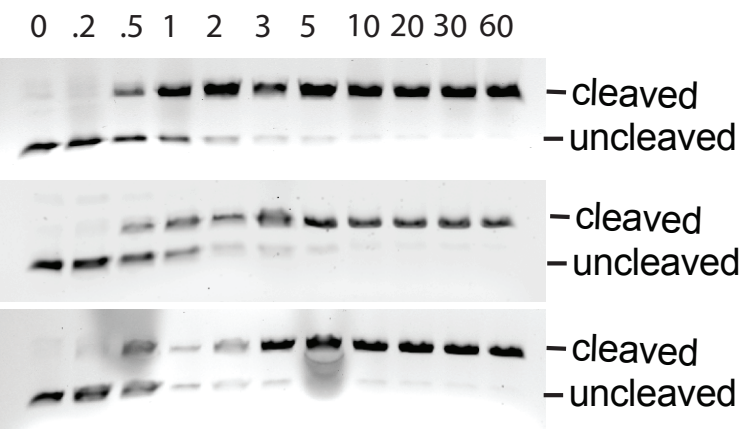

G685A-20mer guide

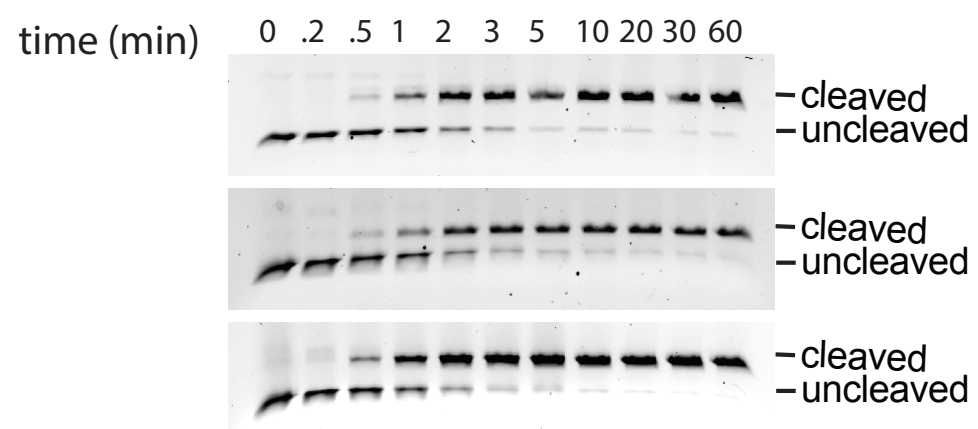

V709A-24mer guide

time (sec) $\quad 0 \quad 2 \quad 5101520253060120300$

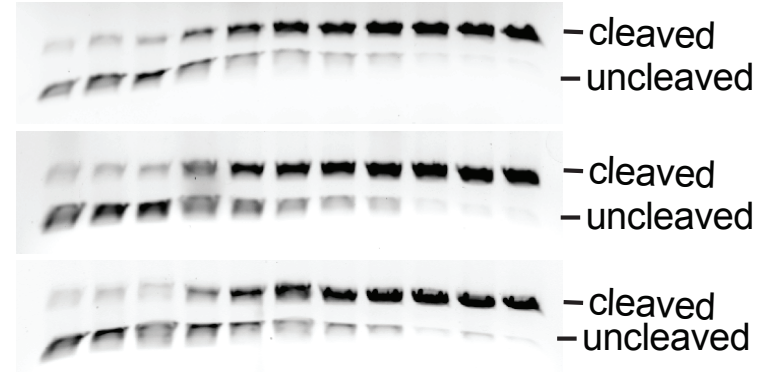

G685A-24mer guide

time (sec) $\quad 0 \quad 2 \quad 5101520253060120300$

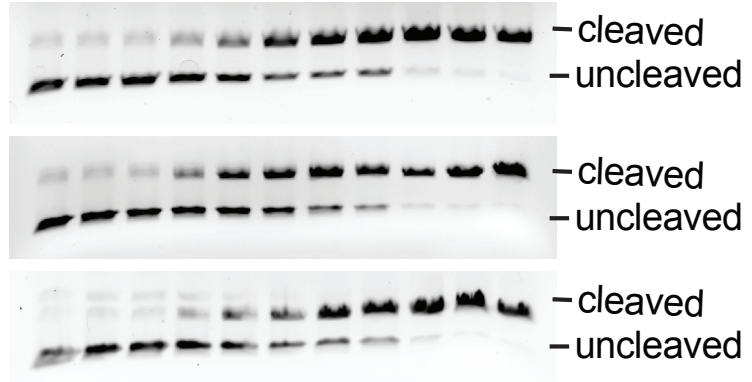

Supplemental Figure 4: Single turnover cleavage assay gel images that were used to produce binding curves in Figure 2. Molar excess of AceCECas9 with either 20mer guide or 24mer guide RNA were incubated with plasmid DNA for increasing time points. The cleavage products were resolved and visualized on agarose gels. 


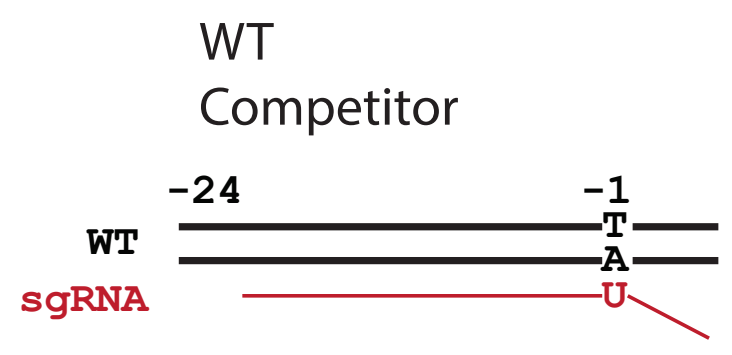

Mismatch

Competitor

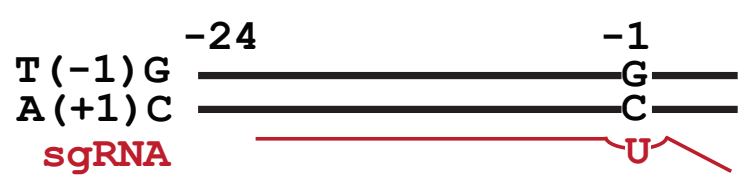

Buldged

Competitor

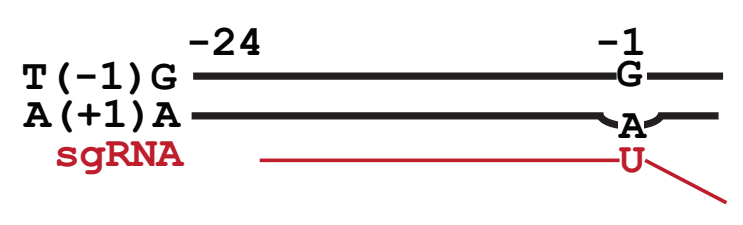

Buldged \& Mismatch

\section{Competitor}

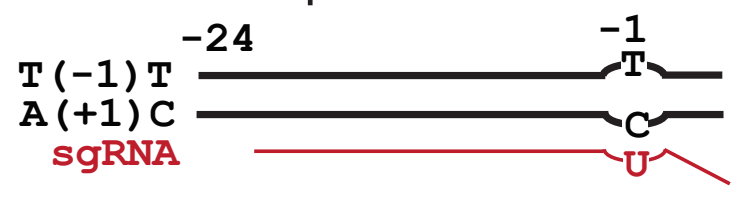

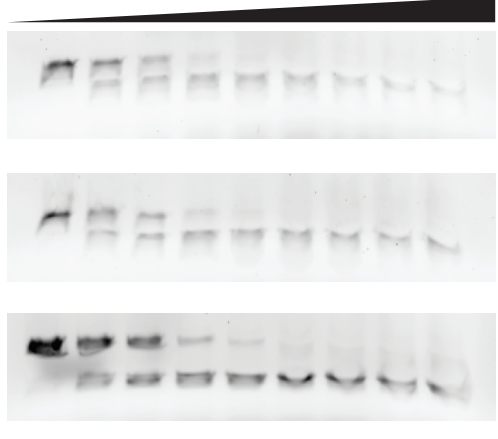

AceCas9

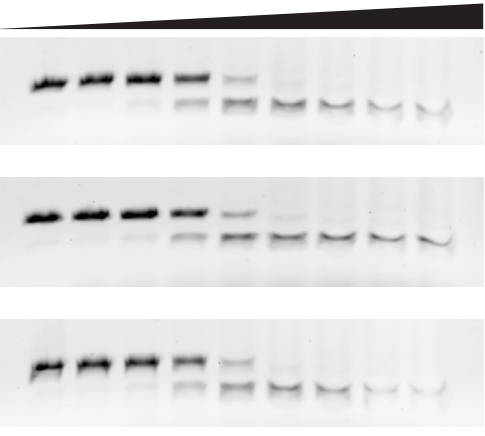

AceCas9
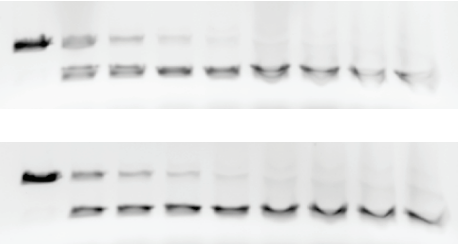

ーーニ

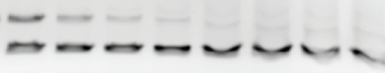

AceCas9
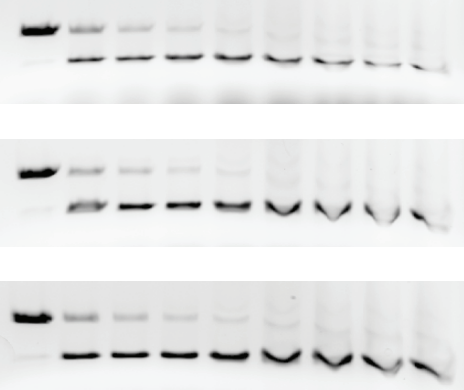

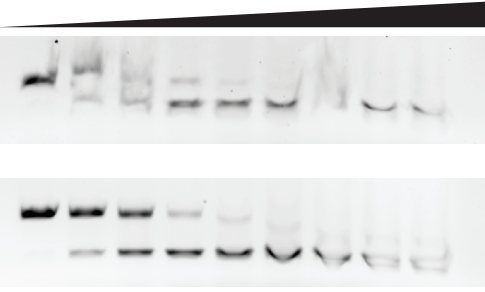

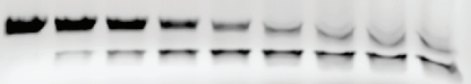

AceCECas9

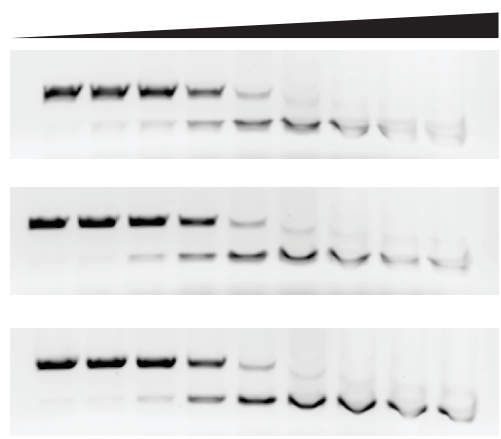

AceCECas9
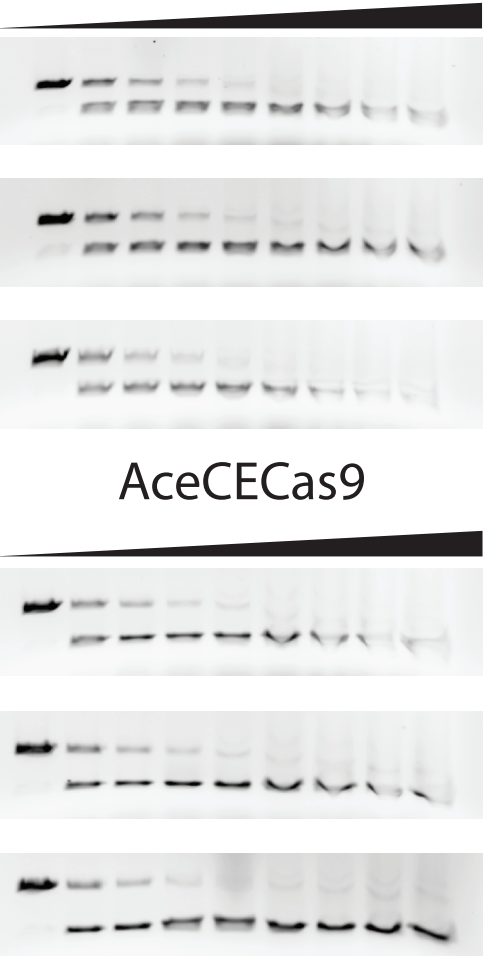

Supplemental Figure 5: Compeition binding gel images that were used to produce the binding curves in Figure 3. Molar excess of the catalytically enhanced (CE) AceCas9, V709A, with the 24mer guide RNA incubated with plasmid substrate DNA were in the presence of increasing concentrations of dsDNA oligo competitors $(0-2.5 \mu \mathrm{M})$ for 15 minutes at $50{ }^{\circ} \mathrm{C}$. Triplicates of the cleavage proucts were resolved, visualized on agarose gels and averaged for curve fitting. 
A bioRxiv preprint doi: https://doi.org/10.1101/2020.07.01.183194; this version posted July 1, 2020. The copyright holder for this preprint (which

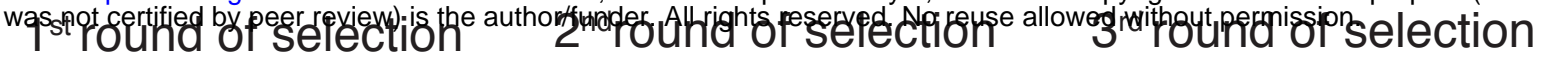
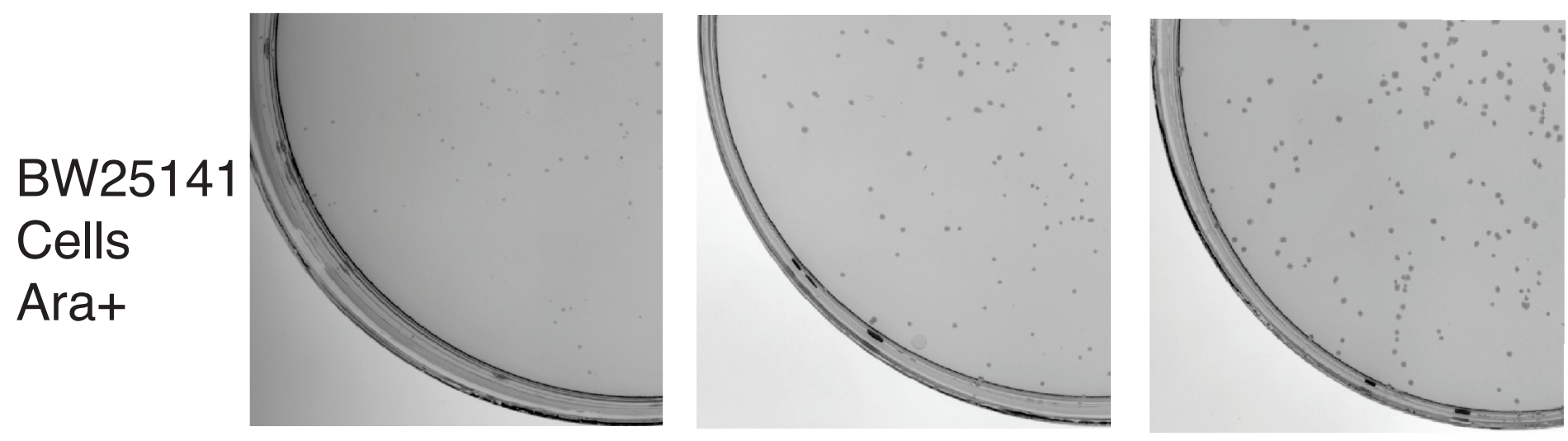

B

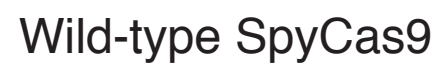

\section{SpyCECas9}

BW25141

Cells

Ara-

BW25141

Cells

Ara+
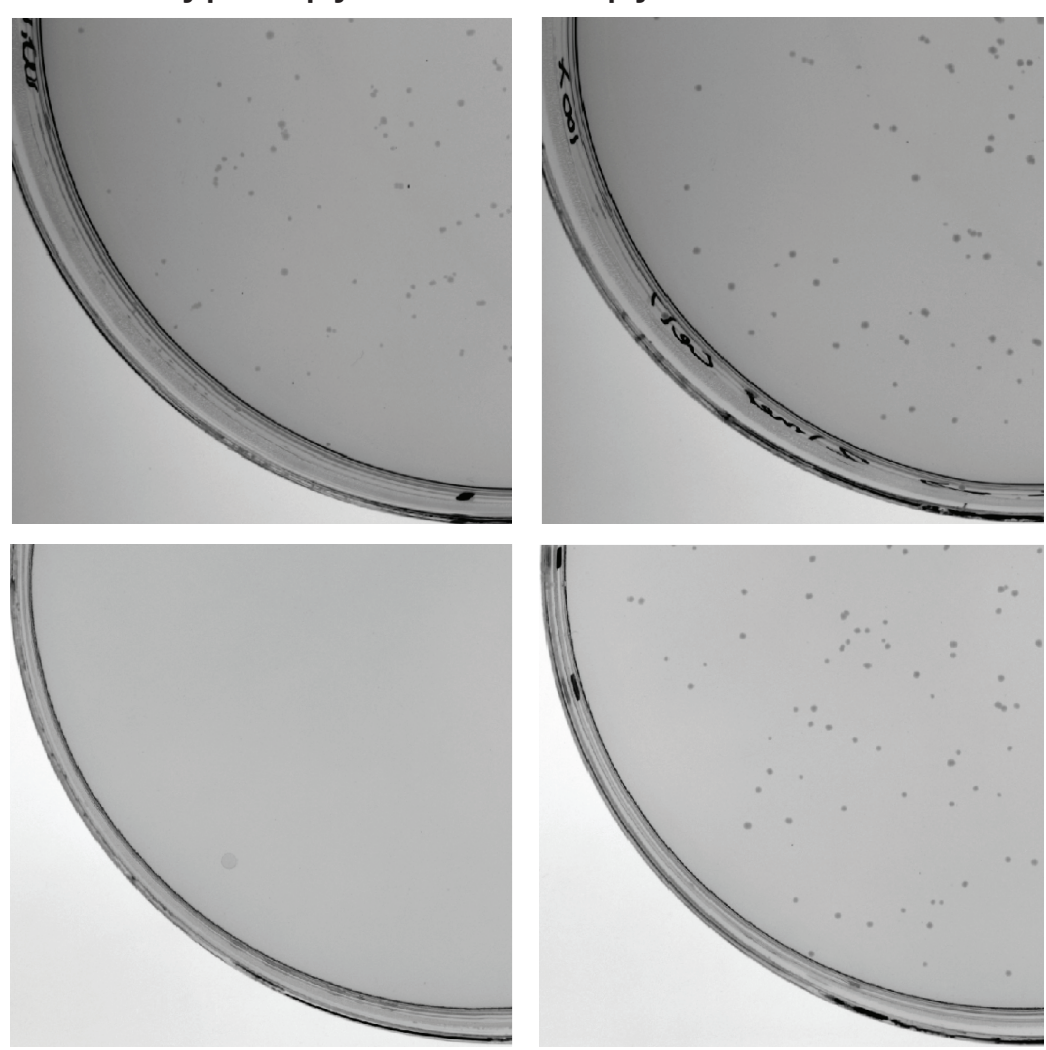

Figure S6. Directed protein evoution of catalytically enhanced SpyCas9 (SpyCECas9). A. $0.1 \mu \mathrm{g}$ DNA plasmid encoding a library of SpyCas9 containing variants at its hinge helices were transformed into BW25141 cells harbouring ccdB-encoding plasmids that bares a 17 mer protospacer for SpyCas9. The entire recovery volume was plated on arbinose-containing (Ara+) plates. Images show the plates for the three rounds of selections. B. Confirmation of the selected SpyCECas9 variants by the cell toxicity assay. The wild-type or the plamsid encoding the catalytically enhanced SpyCas9 was transformed into BW25141 cells harboring the ccdB-encoding plasmid that bears a 17mer protospacer for SpyCas9. Plates contain either no (Ara-) or arabinose (Ara+). 

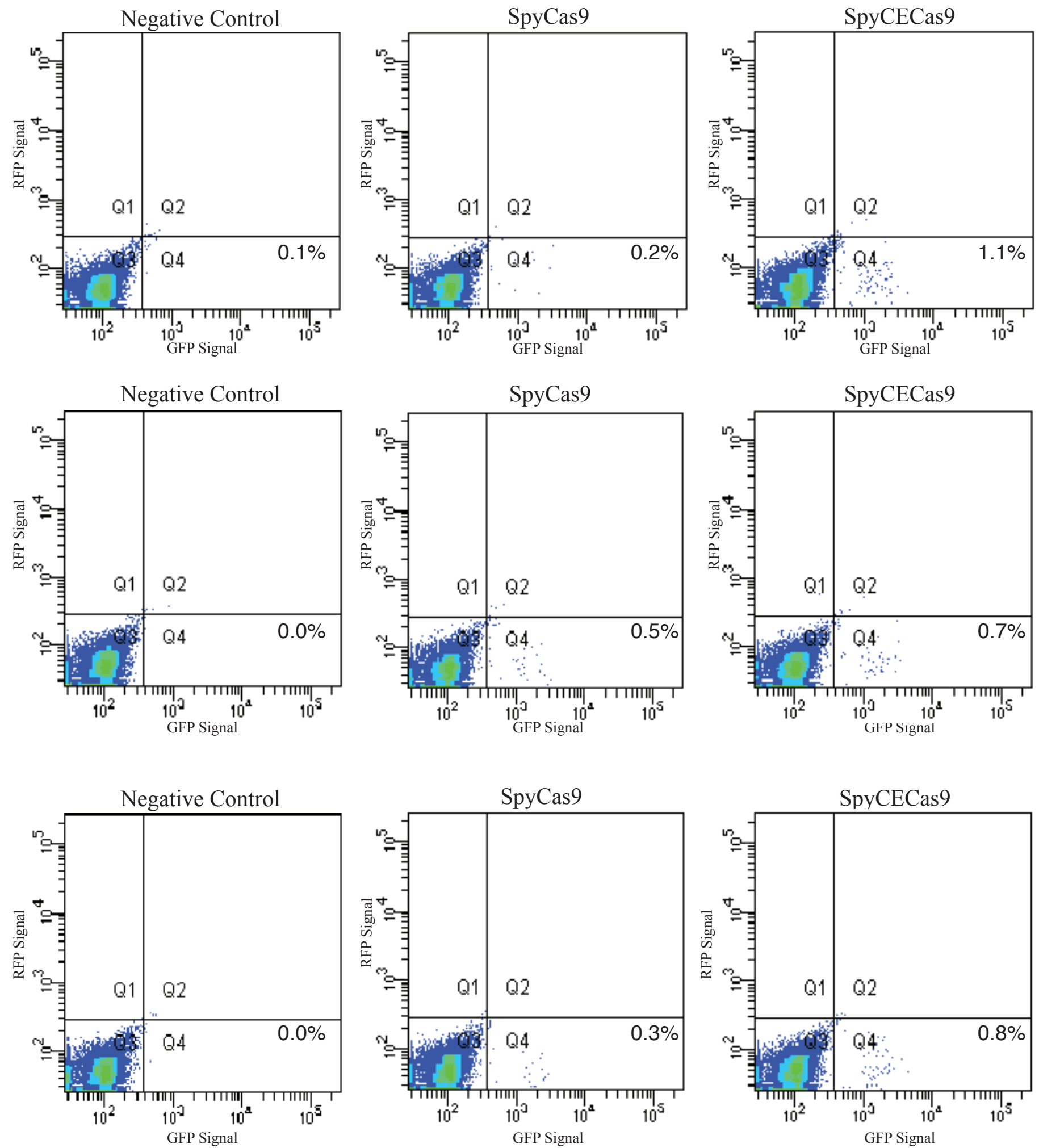

Figure S7. Flow cytometry counts for cells expressing green fluorescent protein (GFP). A. Triplicate experiments of SpyCas9-mediated homology directed repair (HDR) to incorporate GFP-encoding sequence into the LMNB1 gene. Flow cytometry plots displaying GFP fluorecence in cells (x-axis, Q4) following 48-hour post-transfection of the wild-type (SpyCas9) or the catalytically enhanced SpyCas9 (SpyCECas9). The negative control is obtained from cells transfected with the plasmid encoding SpyCas9 (without sgRNA) only. 